\title{
Antibiotic Bioaccumulation in Zooplankton from the Yelang Lake Reservoir of Anshun City, Southwest China
}

\author{
Ni Yan ${ }^{1,3 \#}$, Shengxing Long1,2\#, Kangning Xiong ${ }^{1 *}$,Ting Zhang ${ }^{4}$ \\ ${ }^{1}$ School of Karst Science, Guizhou Normal University, State Engineering Technology Institute \\ for Karst Desertfication Control., Guiyang 550000, Guizhou China \\ ${ }^{2}$ College of Urban and Environmental Sciences, Peking University, Beijing 100871, China \\ ${ }^{3}$ School of Life Science, Guizhou Normal University, Guiyang 550000, Guizhou China
}

${ }^{4}$ Guizhou Provincial Key Laboratory for Rare Animal and Economic Insects of the Mountainous Region, Guizhou Provincial Engineering Research Center for Biological Resources Protection and Efficent Utilization of the Mountainous Region, College of Biology and Environmental Engineering, Guiyang University, Guiyang 550005, Guizhou China

Received: 26 August 2021

Accepted: 6 November 2021

\begin{abstract}

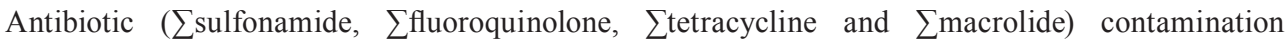
in aquatic systems has remained a global concern in recent years. In this study, fourteen antibiotics, sulfadiazine (SDZ), sulphapyridine (SPD), sulfacetamide (SCT), sulfamethoxazole (SMX), sulfamethazine (SMZ), trimethoprim (TMP), ofloxacin (OFX), lomefloxacin (LFX), ciprofloxacin (CFX), norfloxacin (NFX), oxytetracycline (OTC), tetracycline (TC), dehydroerythromycin (ETM-H2O) and roxithromycin (RTM), were analysed in zooplankton collected from the Yelang Lake Reservoir between May 2016 and Mar. 2017. Our results demonstrated that the antibiotic TC (mean $95.80 \mathrm{ng}$ $\mathrm{g}^{-1}$ ), OTC (mean $167.0 \mathrm{ng} \mathrm{g}^{-1}$ ) and RTM (mean $151.80 \mathrm{ng} \mathrm{g}^{-1}$ ) concentrations were significantly higher in zooplankton than the other antibiotics. TC, OTC and RTM accumulated intensively in zooplankton, and the BAFs of OTC, RTM and TC were significantly higher, with mean BAF values of 78.43, 108.4 and 110.1, respectively. Antibiotic concentrations were strongly correlated with zooplankton phytoplankton biomass, OTC, RTM and SMX concentrations in zooplankton were significantly positively correlated with bacillariophyte biomass, and SDZ and SMZ concentrations in zooplankton were significantly positively correlated with $T$. brevifurcatus, $T$. mongolicus, and $D$. pulex biomass. SPD with P. tunguidus, C. cornuta, TMP, ETM and OFX with P. tunguidus, C. cornuta, M. leuckarti, C. vicinus vicinus, CFX with D. pulex, LFX with T. brevifurcatus, T. mongolicus, Bosmina longirostris, and D. dubium were significantly positively correlated. These results show that bioaccumulation or scavenging of antibiotics across trophic levels is a fundamental and complex component of antibiotic cycling in aquatic environments.
\end{abstract}

Keywords: bioaccumulation, zooplankton, antibiotics, Yelang Lake Reservoir

\footnotetext{
\#These authors contributed equally to this work.

*e-mail: Xiongkn@163.com
} 


\section{Introduction}

The accelerating growth of urban aquatic environments in developing countries, combined with poor control of wastewater and uncontrolled increases in industrial and agricultural activities, have raised serious concerns about pollutants such as heavy metals and antibiotics [1, 2]. Heavy metals are natural constituents of the environment; however, heavy metal pollution in environments has been associated with various human activities (e.g., agrochemical use, urban expansion, and particularly petroleum extraction) $[3,4]$. Most heavy pollutants are transported by runoff to rivers and finally deposited in water bodies with low hydrological dynamics [5]. In addition, antibiotics are extensively used in the treatment of infectious diseases in humans and animals and are also incorporated into animal feed to improve growth [6]. Antibiotics are a class of secondary metabolites produced by microorganisms, as well as chemically synthesized or semi-synthesized analogous compounds, which can inhibit the growth and survival of other microorganisms [7]. Antibiotics are probably the most successful family of drugs to treat microbial infections in humans and animals with specific action on the target. However, antibiotics not only affect the target population but also influence the nontarget population with high toxicity impact [8]. Antibiotics have been detected in aquatic environments such as lakes, rivers, water reservoirs, wastewater influents and effluents, groundwater and even drinking water even though drinking water is treated [9]. Antibiotics can enter the water and land environment through various pathways, such as the discharge of municipal sewage, the manufacturing industry, animal husbandry, landfill leachates of antibiotic disposal, and runoff from agricultural fields containing livestock manure, aquaculture ponds, and urban centres [7]. The abuse of antibiotics has led to higher levels of antibiotic residue, and this is evidence that drugresistant bacteria are evolving, facilitating infections that are difficult to treat [10]. Evidence has shown that the consumption and production of antibiotics in China are the highest in the world [11]. Prescriptions including antibiotics account for $70 \%$ and approximately $30 \%$ in western countries, and annual per capita consumption reaching $138 \mathrm{~g}$ is approximately 2 times the annual per capita consumption of Europe and 10 times the annual per capita consumption of the United States [12-13]. The production of antibiotics in 2013 was up to $2.4810^{5} \mathrm{t}$, which had almost tripled since 2009, and the usage of antibiotics in 2013 was up to $1.6210^{5} \mathrm{t}$, with antibiotics of $5.010^{4} \mathrm{t} / \mathrm{yr}$ into the water and soil environment [14]. Most antibiotics cannot be completely removed by adsorption or metabolism, and a considerable fraction are released into aquatic environments [15]. The concentrations and detection frequencies of antibiotics in aquatic environments in China [16] could lead to selective pressure on water bacteria and induce the formation of antibiotic-resistant bacteria, reducing the therapeutic potential of the antibiotics against human and animal pathogens [16-17]. Therefore, antibiotic residues in aquatic environments could pose a potential threat to the environment and human health [13].

Antibiotics, sulfonamides (SAs), fluoroquinolones (FQs), tetracyclines (TCs) and macrolides (MCs) have been detected at concentrations up to micrograms per litre $\left(\mu \mathrm{g} \cdot \mathrm{L}^{-1}\right)$ in surface waters and nanograms per gram $\left(\mathrm{ng} \cdot \mathrm{g}^{-1}\right)$ in sediments [18-20]. A previous study showed that sulfonamides and fluoroquinolones are the main contamination factors, with the concentrations of SAs ranging from $\mathrm{ND} 940 \mathrm{ng} \mathrm{L^{-1 }}$ and FQs ranging from ND 713.6 ng $\mathrm{L}^{-1}$ in surface lakes [13]. TCs constitute one of the most extensively used antibiotic classes due to their low cost, ease of use and relatively minor side effects [21]. TCs are also the most widely used veterinary drugs and feed additives in the aquaculture and livestock industries of China, with concentrations of ND 87.9 ng L-1 and an average $43.2 \mathrm{ng} \mathrm{L}^{-1}$ in lakes [13]. In China, TCs are produced and consumed on a large scale; for example, the export of TCs was $1.3410^{7} \mathrm{t}$ in 2008 [22], TCs were added at the subtherapeutic level to animal feed to prevent infection and act as growth promoters, and the usage of tetracycline reached $9413 \mathrm{t}$ in 1999 [23]. Evidence has shown that the concentrations of erythromycin (ERM) and roxithromycin (ROM) are relatively high among $\mathrm{MCs}$, with maximum concentrations of $624.8 \mathrm{ng} \mathrm{L}^{-1}$ and $218.3 \mathrm{ng} \mathrm{L}^{-1}$ in Taihu Lake. Exposure to MCs in the aquatic environment of China is similar to the exposure in other surface water bodies. MCs are widely used in concentrated doses to animals and humans, for reasons such as respiratory diseases, intestinal infections and mastitis [24].

In the past few decades, most reservoirs in Guizhou have been involved in aquaculture. Antibiotics have been widely used for disease prevention in fish, shrimp and other aquatic products [25]. Obviously, antibiotics widely used in cage culture are deposited in reservoirs [26]. However, many studies have paid attention to heavy metals in high-load water and residual antibiotics in zooplankton. Therefore, the present study will analyse the enrichment of compound pollutants of antibiotics by zooplankton in the Yelang Lake Reservoir, Guizhou Province, and combine this enrichment with environmental physical and chemical factors in water. Additionally, this study will reveal the effect of eutrophication on polluting antibiotics in zooplankton.

\section{Materials and Methods}

\section{Study Area}

The Yelang Lake Reservoir, Guizhou Province, is located in Puding County, west of central Guizhou, and belongs to the Sancha River drainage basin. The Yelang Lake Reservoir originates from Weining 
County, Bijie, and enters the territory of Guizhou from Yanjiao County, Liuzhi Special Zone, Liupanshui. The county is divided into northern and southern parts by this river from west to east, and the river flows into Pingba County through the Lesna Bridge in the northeast. The length of the river is $67.05 \mathrm{~km}$. The width varies from $35 \mathrm{~m}-75 \mathrm{~m}$, and the water surface area is $22.5 \mathrm{~km}^{2}$. The drainage area in the country is $1069.7645 \mathrm{~km}^{2}$, and the drainage area outside the country is $4854.907 \mathrm{~km}^{2}$. The average annual flow rate is $130.1 \mathrm{~m}^{3} / \mathrm{s}$, and the low water flow is $72.86 \mathrm{~m}^{3} / \mathrm{s}$. The drainage area of Yelang Lake is $5871 \mathrm{~km}^{2}$, accounting for $80.8 \%$ of the drainage area of the Sancha River. The length of the river above the dam is 240 $\mathrm{km}$, and the water surface area is $21.6 \mathrm{~km}^{2}$. The length of the reservoir is $42 \mathrm{~km}$. The widest part of the lake is $2.5 \mathrm{~km}$, and the narrowest part is $60 \mathrm{~m}$. The total water storage is $4.210^{8} \mathrm{~m}^{3}$, and the normal water storage is $3.77 \times 10^{8} \mathrm{~m}^{3}$. The Yelang Lake Reservoir has the functions of aquaculture, drinking water, etc. [27]. According to the geographical environmental characteristics of the research area, a total of 6 sampling sections (Fig. 1) were set up in the Yelang Lake Reservoir.

\section{Water and Phytoplankton Collecting}

Water and phytoplankton were gathered from May 2016 to March 2017. Several indices, including pH value, total nitrogen (TN), and total phosphorus (TP), were described in a previously published study [28]. Dissolved oxygen (DO) and $\mathrm{pH}$ were measured when each sample was collected using a 6600 multisensor sonde (Yellow Springs Inc., Yellow Springs, OH,
USA). Approximately $500 \mathrm{~mL}$ of combined water sample was used for the analysis of chemical oxygen demand $\left(\mathrm{COD}_{\mathrm{Mn}}\right)$, total nitrogen $(\mathrm{TN})$, nitrate $\left(\mathrm{NO}_{3}-\mathrm{N}\right)$, nitrite $\left(\mathrm{NO}_{2}-\mathrm{N}\right)$, ammonia nitrogen $\left(\mathrm{NH}_{3}-\mathrm{N}\right)$, total phosphorus (TP), and phosphate $\left(\mathrm{PO}_{4}-\mathrm{P}\right)$ according to China's national environmental quality standard for surface water (GB 3838-2002). For example, TN and TP concentrations in each sample were determined using the alkaline potassium persulfate oxidation method [29-30].

Water samples for antibiotics were taken from the freezer and thawed. Duplicate aliquots of $10 \mathrm{ml}$ of each sample were transferred to two plastic tubes of $30 \mathrm{ml}$ each. To both aliquots, $10 \mu \mathrm{l}$ of a mixture of internal standard solution $(500 \mu \mathrm{g} / \mathrm{L})$ was added. To one aliquot, $100 \mu \mathrm{l}$ of a mixture of all antibiotics $(2.5 \mu \mathrm{g} / \mathrm{L}$ for the sulfonamides and $10 \mu \mathrm{g} / \mathrm{L}$ for the tetracyclines, quinolones and macrolides) was added. The samples were mixed, and $4 \mathrm{ml}$ of Mcllvain buffer $(0.1 \mathrm{M}$ citric acid, $0.2 \mathrm{M}$ phosphate buffer and Na2EDTA; pH 4) was added. The samples were horizontally shaken for $5 \mathrm{~min}$ at $120 \mathrm{rpm}$, followed by centrifugation at $4000 \mathrm{~g}$ for $10 \mathrm{~min}$. Two hundred milligrams of Strata-X (Phenomenex, USA) SPE columns were washed with $5 \mathrm{ml}$ methanol $(\mathrm{MeOH})$, followed by $5 \mathrm{ml}$ McIlvain buffer. Thereafter, the sample extract was loaded onto the column, followed by a washing step with $5 \mathrm{ml}$ purified water (Milli-Q, Merck, USA). Vacuum pressure was applied to extract the liquid from the SPE columns. Then, the columns were eluted with $5 \mathrm{ml}$ of $\mathrm{MeOH}$, and the eluting liquid was collected in clean collection glass tubes. The collection tubes were placed in a nitrogen evaporator at $40^{\circ} \mathrm{C}$ to evaporate the $\mathrm{MeOH}$. After complete evaporation, the samples were redissolved

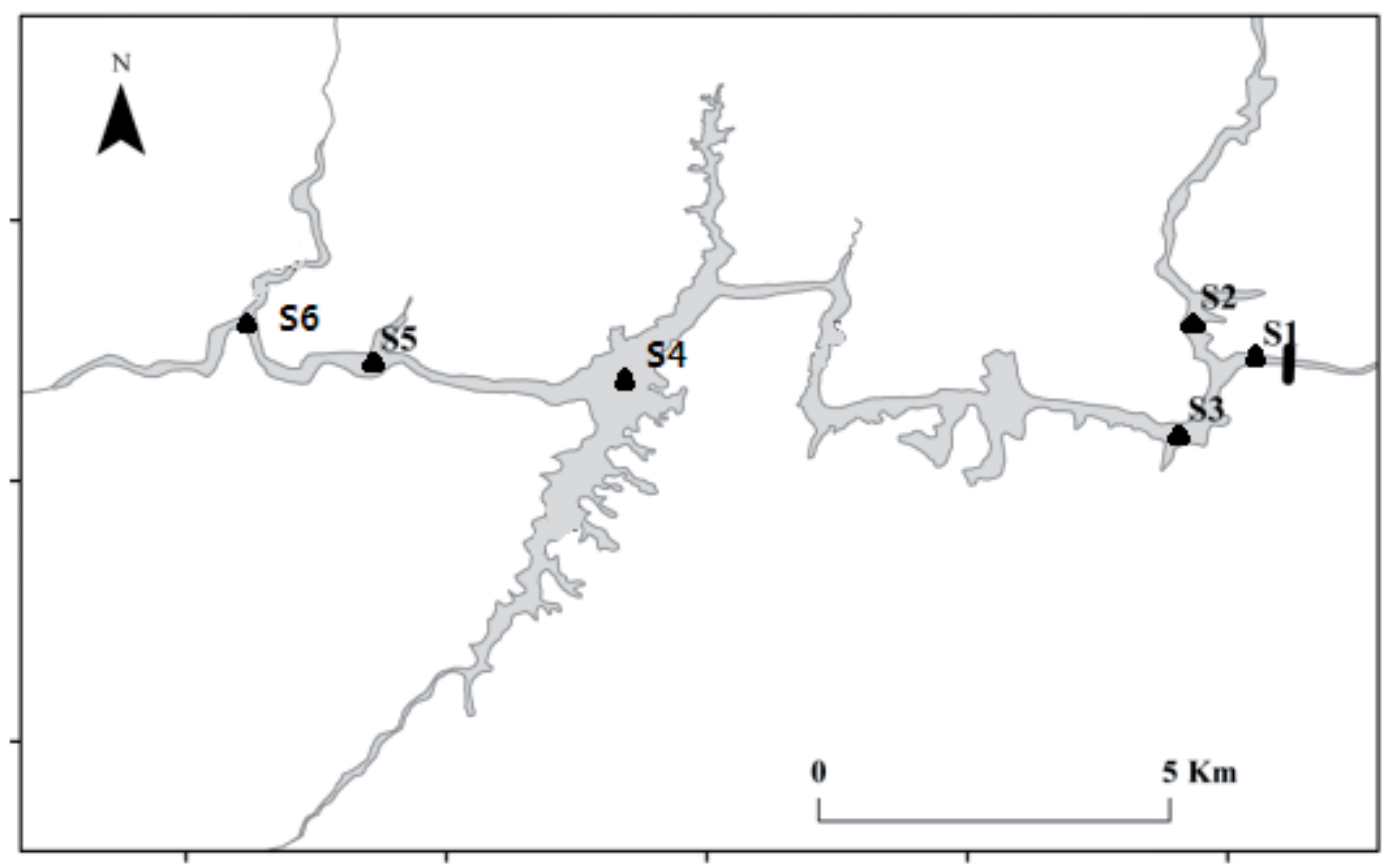

Fig. 1. Sampling collected in Yelang Lake Reservoir of Guizhou Province, South China. 
in $100 \mu \mathrm{l}$ of $\mathrm{MeOH}$ and vortexed for $5 \mathrm{~s}$. Finally, $400 \mu \mathrm{l}$ of purified water was added and vortexed for another $5 \mathrm{~s}$. The homogenized samples were transferred to LCs vials with inserts. The vials were stored at $-20^{\circ} \mathrm{C}$ until further analysis using LC-MS/MS [31].

Plankton samples were collected from surface waters at each sampling site and analysed for phytoplankton community composition, abundance and biomass. Each phytoplankton sample was preserved with the addition of Lugol's iodine, allowed to settle for $48 \mathrm{~h}$ and concentrated to a final volume of $30 \mathrm{~mL}$. Cell density was measured using a Sedgwick-Rafter counting chamber with counts at magnifications between $\sim 200 \AA$ and $\sim 400 \AA$. Species were identified as described by $\mathrm{Hu}$ et al. [32]. Total algal biovolumes for each species were calculated from the number of cells and measured cell sizes. Biomass was determined by converting the cell shapes into volumes and then converting to biomass assuming that $1 \mathrm{~mm}^{3}$ was equivalent to $1 \mathrm{mg}$ fresh weight biomass $[33,34]$. The collection and treatment of antibiotics in water were performed according to a previously published study [28].

\section{Zooplankton Collection, Antibiotics Analyses}

Zooplankton collection and its biomass amount were calculated and described in a previous study [30]. Zooplankton were collected from multiple tows using a nylon mesh net from $0.5 \mathrm{~m}$ above the bottom to the surface at each sampling site [28]. Each tow was put into a 20-L sample bottle; subsequent tows at each site were repeated until the zooplankton dry weight was estimated to be N3.0 g. To clean the sample, the bottle was static for 20 min to concentrate and remove particulate matter from the bottom of the jars. Zooplankton and phytoplankton in the suspended water were transferred to another sample bottle $(20 \mathrm{~L})$, and formaldehyde reagent was added. The fixed (dead) zooplankton were then concentrated at the bottom of the jar; the remaining suspended phytoplankton and soluble organic matter in the supernatant were removed. The zooplankton were transferred into a $100-\mathrm{mL}$ measuring cylinder and thoroughly rinsed with Milli-Q water to remove other impurities. Again, the zooplankton sample was settled for $20 \mathrm{~min}$, and the supernatant was removed. This further removed dead phytoplankton and other impurities. The zooplankton sample was then transferred into sealed 50-m EP tubes and placed in an ice box. Subsequently, the samples were frozen at $-80^{\circ} \mathrm{C}$ in polypropylene and polystyrol containers and stored in a vacuum desiccator prior to analysis [28]. Copepoda, cladocera and rotifera were distinguished based on Wang [35], Jiang and Chu [36] and Shen and Song [37]. The determination of antibiotics from zooplankton and the statistical methods were performed according to a previous study [30]. Pharmaceuticals in the sediment and biota samples were extracted by methanol/acetone
(1:1) using an ASE 350 pressurized liquid extraction system (Dionex, Sunnyvale, USA), followed by purification with an Oasis HLB cartridge. The target pharmaceuticals were analysed using an Agilent 1290 ultrahigh-performance liquid chromatograph coupled with an Agilent 6460 triple quadrupole MS (Agilent, California, USA).

\section{Statistical Methods}

Statistical analyses of the data were performed using SPSS 18.0 (PASW) and Origin 7.5 software. Correlation coefficients ( $\mathrm{r}$ values) and probabilities ( $\mathrm{p}$ values) were calculated for linear regressions comparing variable interactions. F-values were used to indicate the extent of differences between data for samples from different sites. Differences were classified as significant at the $p<0.05$ level. Bioaccumulation factors (BAFs) were calculated using the equation BAF (antibiotic concentration in zooplankton)/(antibiotic concentration in water).

\section{Results and Analysis}

\section{Physical and Chemical Characteristics}

Physicochemical parameters, including dissolved oxygen (DO), $\mathrm{pH}$, chemical oxygen demand $\left(\mathrm{COD}_{\mathrm{Mn}}\right)$, total nitrogen $(\mathrm{TN})$, nitrate $\left(\mathrm{NO}_{3}-\mathrm{N}\right)$, nitrite $\left(\mathrm{NO}_{2}-\mathrm{N}\right)$, ammonia nitrogen $\left(\mathrm{NH}_{3}-\mathrm{N}\right)$, total phosphorus (TP), and phosphate $\left(\mathrm{PO}_{4}-\mathrm{P}\right)$, were measured in the Yelang Lake Reservoir using samples collected from May 2016 to Mar. 2017. The $\mathrm{COD}_{\mathrm{Mn}}, \mathrm{NO}_{3}-\mathrm{N}, \mathrm{NH}_{3}-\mathrm{N}, \mathrm{TP}$, and $\mathrm{PO}_{4}-\mathrm{P}$ concentrations were significant at different sites and at different sampling times and are presented in Table 1. There was a positive correlation between TP and $\mathrm{PO}_{4}-\mathrm{P}$ $(P<0.005, F=137.1)$, and the TP content increased as TN increased $(P<0.005, F=9.16)$. Dissolved oxygen levels in the Yelang Lake Reservoir were consistently higher, and there were no significant changes, indicating that the water was well mixed throughout the year, with mean levels of 7.04- $7.40 \mathrm{mg} \mathrm{L}^{-1}$. Yelang Lake Reservoir was alkaline with a mean $\mathrm{pH}$ value of 7.7. Total phosphorus (TP) concentrations ranged from 0.002 to $0.11 \mathrm{mg} \mathrm{L}^{-1}$, with a mean of $0.02 \mathrm{mg} \mathrm{L}^{-1}$. The Yelang Lake Reservoir was eutrophic according to the Environment Canada trophic status assessment criteria [38] when only TP concentrations were considered, and $\mathrm{PO}_{4}-\mathrm{P}$ ranged from $0.001 \mathrm{mg} \mathrm{L}^{-1}$ to $0.07 \mathrm{mg} \mathrm{L}^{-1}$, with a mean of $0.01 \mathrm{mg} \mathrm{L}^{-1}$. The mean TN concentration of $3.02 \mathrm{mg} \mathrm{L}^{-1}$ in the present study was greater than the limited value of $2.0 \mathrm{mg} \mathrm{L}^{-1}$ and was proposed by the class $\mathrm{V}$ standard for surface water environmental quality (GB3838-2002). The TN concentrations were significant in diverse seasons. $\mathrm{NO}_{3}-\mathrm{N}, \mathrm{NO}_{2}-\mathrm{N}$ and $\mathrm{NH}_{3}-\mathrm{N}$ concentrations ranged from 0.30 to $1.59 \mathrm{mg} \mathrm{L}^{-1}, 0.01$ to $0.10 \mathrm{mg} \mathrm{L}^{-1}$ and 0.01 to $0.98 \mathrm{mg} \mathrm{L}^{-1}$, with means of $1.07 \mathrm{mg} \mathrm{L}^{-1}, 0.023 \mathrm{mg} \mathrm{L}^{-1}$ 
and $0.25 \mathrm{mg} \mathrm{L} \mathrm{L}^{-1}$, respectively. $\mathrm{COD}_{\mathrm{Mn}}$ concentration ranged from 3.10 to $10.90 \mathrm{mg} \mathrm{L}^{-1}$, with a mean of $6.31 \mathrm{mg} \mathrm{L}^{-1}$ (Table 1).
Antibiotics concentrations are listed in Table 2. According to Table 2, the antibiotic concentrations were trace amounts, and the higher concentration did

Table 1. Physical and chemical parameters in the in Yelang Lake Reservoir.

\begin{tabular}{|c|c|c|c|c|c|c|c|c|c|c|}
\hline Times & Sites & $\begin{array}{c}\mathrm{TP} \\
\mathrm{mg} \mathrm{L}^{-1}\end{array}$ & $\begin{array}{l}\mathrm{PO}_{4}-\mathrm{P} \\
\mathrm{mg} \mathrm{L}^{-1}\end{array}$ & $\begin{array}{c}\mathrm{TN} \\
\mathrm{mg} \mathrm{L}^{-1}\end{array}$ & $\begin{array}{l}\mathrm{NO}_{3}-\mathrm{N} \\
\mathrm{mg} \mathrm{L}^{-1}\end{array}$ & $\begin{array}{l}\mathrm{NH}_{3}-\mathrm{N} \\
\mathrm{mg} \mathrm{L}^{-1}\end{array}$ & $\begin{array}{l}\mathrm{NO}_{2}-\mathrm{N} \\
\mathrm{mg} \mathrm{L}^{-1}\end{array}$ & $\begin{array}{l}\mathrm{COD}_{\mathrm{Mn}} \\
\mathrm{mg} \mathrm{L}^{-1}\end{array}$ & $\begin{array}{c}\mathrm{DO} \\
\mathrm{mg} \mathrm{L}^{-1}\end{array}$ & $\mathrm{pH}$ \\
\hline \multirow{6}{*}{ May 2016} & $\mathrm{~S} 1$ & 0.003 & 0.001 & 3.02 & 0.94 & 0.39 & 0.01 & 6.8 & 8.38 & 7.8 \\
\hline & S2 & 0.008 & 0.008 & 3.43 & 1.09 & 0.38 & 0.01 & 6.9 & 8.61 & 7.7 \\
\hline & $\mathrm{S} 3$ & 0.013 & 0.009 & 3.12 & 0.73 & 0.35 & 0.02 & 6.3 & 8.67 & 7.4 \\
\hline & S4 & 0.013 & 0.008 & 2.94 & 0.94 & 0.37 & 0.01 & 8.2 & 8.94 & 7.3 \\
\hline & S5 & 0.012 & 0.011 & 3.48 & 0.89 & 0.98 & 0.02 & 5.3 & 8.79 & 7.8 \\
\hline & S6 & 0.017 & 0.011 & 3.11 & 0.82 & 0.72 & 0.01 & 6.9 & 8.47 & 7.9 \\
\hline \multirow{6}{*}{ Jun. 2016} & $\mathrm{~S} 1$ & 0.020 & 0.012 & 3.21 & 1.54 & 0.56 & 0.01 & 5.8 & 8.51 & 7.9 \\
\hline & $\mathrm{S} 2$ & 0.004 & 0.001 & 3.08 & 1.09 & 0.36 & 0.01 & 6.6 & 6.94 & 7.6 \\
\hline & S3 & 0.006 & 0.005 & 3.53 & 0.96 & 0.35 & 0.01 & 5.0 & 6.4 & 7.6 \\
\hline & S4 & 0.008 & 0.007 & 3.69 & 1.05 & 0.48 & 0.01 & 6.1 & 6.91 & 7.9 \\
\hline & S5 & 0.011 & 0.007 & 3.23 & 0.81 & 0.35 & 0.02 & 6.5 & 8.12 & 7.7 \\
\hline & S6 & 0.012 & 0.010 & 3.54 & 1.04 & 0.91 & 0.02 & 6.0 & 6.89 & 7.8 \\
\hline \multirow{6}{*}{ Aug. 2016} & S1 & 0.021 & 0.011 & 3.58 & 0.99 & 0.65 & 0.02 & 5.2 & 7.12 & 7.9 \\
\hline & S2 & 0.003 & 0.002 & 2.89 & 1.15 & 0.06 & 0.01 & 5.2 & 8.56 & 7.6 \\
\hline & S3 & 0.002 & 0.004 & 2.06 & 1.12 & 0.04 & 0.01 & 8.4 & 8.38 & 7.6 \\
\hline & S4 & 0.038 & 0.020 & 2.30 & 1.08 & 0.01 & 0.05 & 5.5 & 8.03 & 7.9 \\
\hline & S5 & 0.003 & 0.002 & 1.95 & 0.87 & 0.14 & 0.01 & 5.6 & 8.49 & 7.8 \\
\hline & S6 & 0.008 & 0.003 & 1.49 & 0.86 & 0.15 & 0.01 & 7.6 & 8.21 & 7.3 \\
\hline \multirow{6}{*}{ Sept. 2016} & S1 & 0.015 & 0.009 & 1.64 & 1.08 & 0.14 & 0.01 & 6.6 & 8.17 & 7.4 \\
\hline & S2 & 0.052 & 0.025 & 4.02 & 1.13 & 0.09 & 0.07 & 8.9 & 6.12 & 7.6 \\
\hline & S3 & 0.075 & 0.028 & 3.76 & 1.10 & 0.05 & 0.07 & 4.1 & 6.08 & 7.6 \\
\hline & $\mathrm{S} 4$ & 0.106 & 0.041 & 4.19 & 1.12 & 0.07 & 0.08 & 4.0 & 5.69 & 7.6 \\
\hline & S5 & 0.052 & 0.019 & 3.45 & 1.59 & 0.06 & 0.10 & 4.0 & 6.03 & 7.7 \\
\hline & S6 & 0.092 & 0.070 & 2.98 & 0.30 & 0.02 & 0.01 & 3.1 & 6.34 & 7.4 \\
\hline \multirow{6}{*}{ Nov. 2016} & S1 & 0.039 & 0.010 & 3.79 & 1.11 & 0.33 & 0.04 & 10.9 & 6.28 & 8.1 \\
\hline & $\mathrm{S} 2$ & 0.037 & 0.008 & 3.57 & 1.06 & 0.28 & 0.04 & 7.2 & 6.22 & 7.9 \\
\hline & $\mathrm{S} 3$ & 0.019 & 0.003 & 2.84 & 1.00 & 0.20 & 0.03 & 7.2 & 6.33 & 7.8 \\
\hline & S4 & 0.030 & 0.001 & 2.95 & 0.99 & 0.26 & 0.03 & 6.9 & 6.16 & 7.8 \\
\hline & S5 & 0.020 & 0.002 & 3.01 & 0.91 & 0.17 & 0.03 & 8.2 & 6.26 & 7.8 \\
\hline & S6 & 0.017 & 0.007 & 4.33 & 1.04 & 0.23 & 0.03 & 8.0 & 6.0 & 8.0 \\
\hline \multirow{6}{*}{ Dec. 2017} & $\mathrm{~S} 1$ & 0.010 & 0.003 & 2.96 & 1.17 & 0.08 & 0.01 & 8.9 & 6.66 & 7.9 \\
\hline & $\mathrm{S} 2$ & 0.011 & 0.004 & 2.69 & 1.22 & 0.04 & 0.01 & 5.8 & 9.78 & 7.8 \\
\hline & $\mathrm{S} 3$ & 0.018 & 0.005 & 3.42 & 1.20 & 0.03 & 0.01 & 4.2 & 5.98 & 7.9 \\
\hline & $\mathrm{S} 4$ & 0.020 & 0.007 & 3.04 & 0.94 & 0.04 & 0.01 & 9.3 & 7.46 & 7.9 \\
\hline & S5 & 0.015 & 0.005 & 3.12 & 1.21 & 0.09 & 0.01 & 5.4 & 6.64 & 7.8 \\
\hline & S6 & 0.015 & 0.005 & 2.72 & 1.18 & 0.03 & 0.01 & 6.9 & 5.76 & 7.8 \\
\hline
\end{tabular}


Table 1. Continued.

\begin{tabular}{|c|c|c|c|c|c|c|c|c|c|c|}
\hline \multirow{6}{*}{ Feb.2017 } & S1 & 0.019 & 0.007 & 2.95 & 1.41 & 0.05 & 0.01 & 7.1 & 7.29 & 7.8 \\
\hline & $\mathrm{S} 2$ & 0.009 & 0.001 & 2.39 & 1.33 & 0.09 & 0.02 & 4.8 & 7.63 & 7.8 \\
\hline & $\mathrm{S} 3$ & 0.005 & 0.003 & 2.64 & 1.03 & 0.11 & 0.02 & 3.2 & 8.21 & 7.7 \\
\hline & S4 & 0.006 & 0.004 & 2.39 & 1.21 & 0.07 & 0.03 & 3.7 & 7.51 & 7.9 \\
\hline & S5 & 0.020 & 0.014 & 3.05 & 1.03 & 0.07 & 0.02 & 4.0 & 8.23 & 7.7 \\
\hline & S6 & 0.015 & 0.011 & 3.22 & 1.05 & 0.08 & 0.03 & 5.3 & 8.11 & 7.8 \\
\hline \multirow{6}{*}{ Mar.2017 } & S1 & 0.015 & 0.009 & 2.35 & 1.39 & 0.20 & 0.02 & 6.1 & 7.48 & 7.9 \\
\hline & $\mathrm{S} 2$ & 0.015 & 0.011 & 2.84 & 1.30 & 0.22 & 0.02 & 6.6 & 7.31 & 7.7 \\
\hline & $\mathrm{S} 3$ & 0.023 & 0.011 & 2.97 & 0.94 & 0.14 & 0.02 & 5.8 & 7.73 & 7.8 \\
\hline & S4 & 0.017 & 0.013 & 2.94 & 1.23 & 0.22 & 0.02 & 7.0 & 8.11 & 8.0 \\
\hline & S5 & 0.021 & 0.008 & 2.52 & 1.12 & 0.98 & 0.02 & 8.2 & 7.43 & 8.0 \\
\hline & S6 & 0.032 & 0.010 & 2.65 & 1.32 & 0.36 & 0.02 & 7.5 & 8.06 & 7.8 \\
\hline \multicolumn{2}{|c|}{ Min } & 0.002 & 0.001 & 1.49 & 0.30 & 0.01 & 0.01 & 3.10 & 5.69 & 7.3 \\
\hline \multicolumn{2}{|c|}{ Max } & 0.11 & 0.07 & 4.33 & 1.59 & 0.98 & 0.10 & 10.90 & 9.78 & 8.1 \\
\hline \multicolumn{2}{|c|}{ Average } & 0.02 & 0.01 & 3.02 & 1.08 & 0.25 & 0.02 & 6.31 & 7.41 & 7.7 \\
\hline
\end{tabular}

not exceed $6.8 \mathrm{ng} \mathrm{L^{-1 }}$. Mean antibiotic (sulfadiazine (SDZ), sulfapyridine (SPD), sulfacetamide (SCT), sulfamethoxazole (SMX), sulfamethazine (SMZ), trimethoprim (TMP), lomefloxacin (LFX), ciprofloxacin (CFX), norfloxacin (NFX), oxytetracycline (OTC), tetracycline (TC), dehydroerythromycin (ETM$\mathrm{H}_{2} \mathrm{O}$ ), and roxithromycin (RTM) concentrations) concentrations in the water were also distinctly different across the 6 sites of the Yelang Lake reservoir study area, and the mean SPD, SCT, SMX, TMP and SMZ concentrations in the water were higher than the concentrations of other antibiotics at all sampling sites.

\section{Phytoplankton Biomass and Zooplankton Communities}

Fig. 2a) shows that the main phytoplankton in the Yelang Lake Reservoir is Microcystis aeruginosa Kütz. (cyanobacteria); Pediastrum spp., Crucigenia spp., Scenedesmus spp. (Chlorophyceae); Coscinodiscus spp., Cyclotella spp. (sensu lato), Melosira spp. (including Aulacoseira spp.) (diatoms); Peridiniopsis spp. (Dinophyceae); Cryptomonas spp. (Cryptophyceae); Dinobryon spp. (Chrysophyceae); and Euglena spp. (Euglenoidea) (Fig. 2a). The highest biomass was diatom, and its proportion and average value were between 6.0 and $95.10 \%$ and $53.38 \%$, respectively. Successively, the second chief biomass was Dinophyceaen (Peridiniopsis spp.), and its proportion and average

Table 2. Antibiotics concentrations (ng L $\left.{ }^{-1}\right)$ in Yelang Lake Reservoir.

\begin{tabular}{|c|c|c|c|c|c|c|c|c|c|c|c|c|c|c|}
\hline Sites & SDZ & SPD & SCT & SMX & SMZ & TMP & NFX & CFX & LFX & OFX & OTC & TC & ETM- $H_{2} \mathrm{O}$ & RTM \\
\hline S1 & 2.5 & 1.4 & 2.0 & 2.4 & 1.9 & 3.3 & 4.2 & 1.7 & 2.9 & 1.9 & 2.0 & 1.3 & 2.4 & 1.8 \\
\hline S2 & 5.3 & 2.8 & 1.7 & 1.8 & 4.1 & 4.1 & 0.6 & 0.3 & 0.3 & 1.2 & 3.8 & 2.8 & 1.8 & 2.1 \\
\hline S3 & 5.6 & 1.0 & 7.4 & 1.4 & 2.4 & 6.0 & 3.7 & 0.4 & 1.3 & 0.3 & 2.1 & 0.1 & 2.4 & 1.6 \\
\hline S4 & 1.6 & 2.8 & 8.5 & 9.3 & 2.2 & 3.7 & 2.6 & 2.6 & 2.9 & 1.8 & 1.5 & 0.5 & 1.4 & 1.2 \\
\hline S5 & 0.7 & 6.8 & 2.1 & 9.5 & 6.1 & 3.1 & 3.8 & 1.5 & 2.4 & 1.2 & 2.2 & 0.3 & 1.2 & 1.3 \\
\hline S6 & 0.6 & 4.0 & 1.3 & 7.7 & 4.1 & 0.5 & 2.2 & 3.2 & 1.8 & 2.5 & 1.2 & 0.2 & 1.4 & 0.4 \\
\hline Min & 0.6 & 1 & 1.3 & 1.4 & 1.9 & 0.5 & 0.6 & 0.3 & 0.3 & 0.3 & 1.2 & 0.1 & 1.2 & 0.4 \\
\hline Max & 5.6 & 6.8 & 8.5 & 9.5 & 6.1 & 6 & 4.2 & 3.2 & 2.9 & 2.5 & 3.8 & 2.8 & 2.4 & 2.1 \\
\hline Average & 2.72 & 3.13 & 3.83 & 5.35 & 3.47 & 3.45 & 2.85 & 1.62 & 1.93 & 1.48 & 2.13 & 0.87 & 1.77 & 1.40 \\
\hline
\end{tabular}



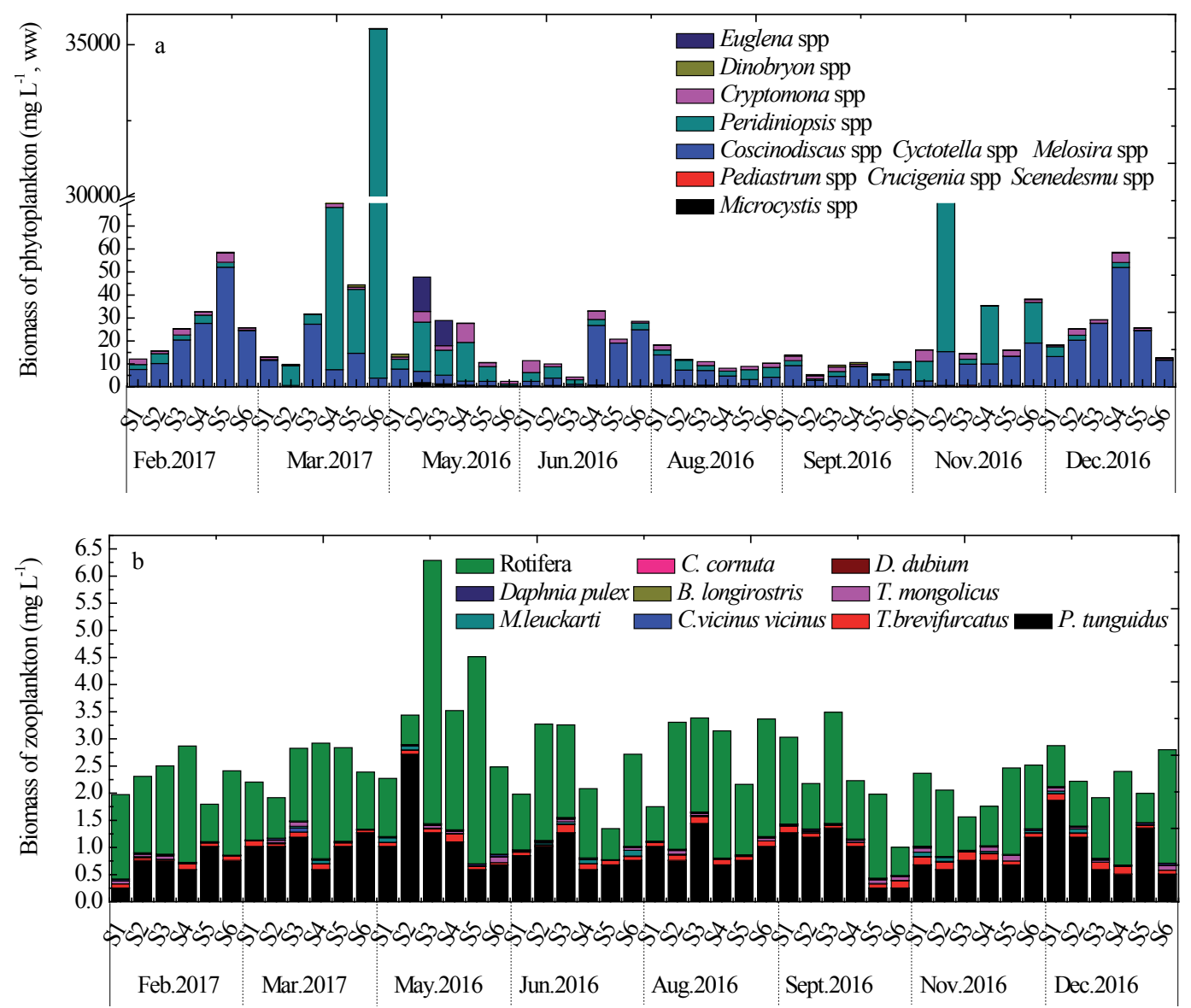

Fig. 2. Phytoplankton a) and zooplankton, b) species biomass in Yelang Lake Reservoir (Guizhou Province, PR China) between May. 2016 and March 2017.

values were $0.11-99.99 \%$ and $30.11 \%$, respectively. The lower biomass was Chlorophytes and M. aeruginosa. The average proportion of Cryptomonas spp. (Cryptophyceae) was $12.45 \%$, ranging from $0.02 \%-53.6 \%$ during May 2016 and Mar. 2017. The other phytoplankton changed greatly during the investigation periods, and the average biomass levels were lower than 2.32\% (Fig. 2a).

Sixteen zooplankton species were found in the Yelang Lake Reservoir study area, including 4 Cladocera, 5 Copepoda, and 7 Rotifera (Table 3). Phyllodiaptomus tunguidus (Shen \& Tai, 1964), Thermocyclops brevifurcatus (Harada, 1931), and Bosmina longirostris (O.F. Müller) were seen frequently during the study period, and their average biomass concentrations were $0.928 \mathrm{mg} \mathrm{L}^{-1}, 0.0871 \mathrm{mg} \mathrm{L}^{-1}$ and $0.0062 \mathrm{mg} \mathrm{L}^{-1}$, respectively. Zooplankton community composition and biomass changed between May 2016 and Mar. 2017. Rotifer biomass was consistently higher than the biomass of other species, and the mean biomass was $1.488 \mathrm{mg} \mathrm{L} \mathrm{L}^{-1}$. P. tunguidus dominated the copepod community, contributing between $12.90 \%$ and $79.01 \%$ of the total zooplankton biomass, with a mean of $37.2 \%$ (Fig. 2b).

\section{Antibiotic Concentrations in Zooplankton}

Antibiotics consisting of six SAs, four FQs, two MLs and two TCs were included in this investigation. Six SAs were sulfadiazine (SDZ), sulfapyridine (SPD), sulfacetamide (SCT), sulfamethoxazole (SMX), sulfamethazine (SMZ) and trimethoprim (TMP). The four FQs were ofloxacin (OFX), lomefloxacin (LFX), ciprofloxacin (CFX) and norfloxacin (NFX). The two TCs were oxytetracycline (OTC) and tetracycline (TC), and the two MLs were dehydroerythromycin (ETM- $\left.\mathrm{H}_{2} \mathrm{O}\right)$ and roxithromycin (RTM). Antibiotic concentrations varied greatly in the zooplankton at different sites, and the mean antibiotic concentrations in order of abundance were $\mathrm{SMX}>\mathrm{S}$ $\mathrm{CT}=\mathrm{SMZ}>\mathrm{SPD}>\mathrm{TMP}>\mathrm{SDZ}>\mathrm{NFX}>\mathrm{LFX}>\mathrm{OTC}>\mathrm{OF}$ $\mathrm{X}>$ ETM $-\mathrm{H}_{2} \mathrm{O}>\mathrm{CFX}>\mathrm{RTM}>\mathrm{TC} \quad($ Table 4). Antibiotic bioaccumulation factors (BAFs) were significantly different in zooplankton. RTM, TC and OTC bioaccumulation factors (BAFs) were significantly higher than BAFs of SPD, SCT, SMX, SMZ and TMP (Table 4). RTM, TC and OTC bioaccumulation factors (BAFs) were significantly higher than SPD, SCT, SMX, SMZ and TMP (Table 4). Furthermore, antibiotics in the zooplankton varied among sample sites. The mean bioaccumulation of NFX, OTC, TC and RTM 
Table 3. Dominant zooplankton species in Yelang Lake Reservoir.

\begin{tabular}{|c|c|}
\hline Date & Dominant species and groups \\
\hline May. 2016 & $\begin{array}{c}\text { Cladocera: Bosmina longirostris, Diaphanosoma dubium. } \\
\text { Copepoda: Phyllodiaptomus tunguidus Shen \& Tai, 1964, Cyclops vicinus vicinus Uljanin, 1875, Thermocyclops } \\
\text { brevifurcatus (Harada, 1931), Themocyclops mongolicus Kiefer 1937. } \\
\text { Rotifera: Asplanchna priodonta, Keratella cochlearis, Filinia longiseta, Brachionus calyciflorua. }\end{array}$ \\
\hline Jun. 2016 & $\begin{array}{c}\text { Cladocera: Bosmina longirostris, Diaphanosoma dubium. } \\
\text { Copepoda: Phyllodiaptomus tunguidus Shen \& Tai, 1964, Thermocyclops brevifurcatus (Harada, 1931), } \\
\text { Themocyclops mongolicus Kiefer } 1937 . \\
\text { Rotifera: Asplanchna priodonta, Keratella cochlearis, Filinia longiseta, Brachionus calyciflorua. }\end{array}$ \\
\hline Aug. 2016 & $\begin{array}{c}\text { Cladocera: Bosmina longirostris, Diaphanosoma dubium. } \\
\text { Copepoda: Phyllodiaptomus tunguidus Shen \& Tai, 1964, Cyclops vicinus vicinus Uljanin, 1875, Themocyclops } \\
\text { mongolicus Kiefer 1937, Thermocyclops brevifurcatus (Harada, 1931). } \\
\text { Rotifera: Asplanchna priodonta, Keratella cochlearis, Filinia longiseta, Brachionus calyciflorua. }\end{array}$ \\
\hline Sept. 2016 & $\begin{array}{c}\text { Cladocera: Bosmina longirostris, Diaphanosoma dubium. } \\
\text { Copepoda: Phyllodiaptomus tunguidus Shen \& Tai, 1964, Cyclops vicinus vicinus Uljanin, 1875, Themocyclops } \\
\text { mongolicus Kiefer 1937, Mesocyclops leuckarti Claus 1857, Thermocyclops brevifurcatus (Harada, 1931). } \\
\text { Rotifera: Asplanchna priodonta, Keratella cochlearis, Filinia longiseta, Brachionus calyciflorua. }\end{array}$ \\
\hline Nov. 2016 & $\begin{array}{c}\text { Cladocera: Bosmina longirostris, Diaphanosoma dubium. } \\
\text { Copepoda: Phyllodiaptomus tunguidus Shen \& Tai, 1964, Cyclops vicinus vicinus Uljanin, 1875, Themocyclops } \\
\text { mongolicus Kiefer 1937, Mesocyclops leuckarti Claus 1857, Thermocyclops brevifurcatus (Harada, 1931). } \\
\text { Rotifera: Asplanchna priodonta, Keratella cochlearis, Filinia longiseta, Brachionus calyciflorua. }\end{array}$ \\
\hline Dec. 2016 & $\begin{array}{c}\text { Cladocera: Bosmina longirostris (O.F. Müller) 1776, Daphnia pulex Leydig 1860, Diaphanosoma dubium } \\
\text { Manuilova } 1964 . \\
\text { Copepoda: Phyllodiaptomus tunguidus Shen \& Tai, 1964, Thermocyclops brevifurcatus (Harada, 1931), Cyclops } \\
\text { vicinus vicinus Uljanin, 1875, Mesocyclops leuckarti Claus 1857, Themocyclops mongolicus Kiefer 1937. } \\
\text { Rotifera: Asplanchna priodonta Gosse 1850, Keratella valga Ahlstrom, 1934, Brachionus calyciflorua Pallas, } \\
\text { 1766, Brachionus angularis Gosse 1851. }\end{array}$ \\
\hline Feb. 2017. & $\begin{array}{r}\text { Cladocera: Bosmina longirostris, Daphnia pulex, Ceriodahnia cornuta Sars } 1885 . \\
\text { Copepoda: Phyllodiaptomus tunguidus Shen \& Tai, 1964, Cyclops vicinus vicinus Uljanin, 1875, Thermocyclops } \\
\text { brevifurcatus (Harada, 1931), Themocyclops mongolicus Kiefer. } \\
\text { Rotifera: Asplanchna priodonta, Keratella cochlearis Gosse 1851, Filinia longiseta Ehrenberg 1834, Brachionus } \\
\text { calyciflorua Pallas 1766, Brachionus angularis. }\end{array}$ \\
\hline Mar. 2017 & $\begin{array}{c}\text { Cladocera: Bosmina longirostris, Daphnia pulex Leydig, Ceriodahnia cornuta } \\
\text { Copepoda: Phyllodiaptomus tunguidus Shen \& Tai, 1964, Cyclops vicinus vicinus Uljanin, 1875, Mesocyclops } \\
\text { leuckarti, Thermocyclops brevifurcatus (Harada, 1931). } \\
\text { Rotifera: Asplanchna priodonta, Keratella valga Ehrenberg 1834, Keratella cochlearis, Polyarthra trigla } \\
\text { Ehrenberg 1834, Brachionus calyciflorua, Brachionus angularis. }\end{array}$ \\
\hline
\end{tabular}

was higher than the mean bioaccumulation of other antibiotics across seasons (Table 4).

Fig. 3 shows the measured concentrations of antibiotics in the Yelang Lake Reservoir. According to two-way ANOVA, spatial variation in the total amount of 14 antibiotics was highly significant in zooplankton $(p<0.001)$. The concentration of $\sum$ antibiotics was defined as the sum concentrations of the target antibiotic of a certain category. Concentrations of $\sum$ sulfonamides, $\sum$ fluoroquinolones, $\sum$ tetracyclines and $\sum$ macrolides ranged from 25.66 to $746.63 \mathrm{ng} \mathrm{g}^{-1}, 15.60$ to $598.83 \mathrm{ng} \mathrm{g}^{-1}, 9.067$ to $1269.07 \mathrm{ng} \mathrm{g}^{-1}$ and 8.19 to $1218.09 \mathrm{ng} \mathrm{g}^{-1}$ in zooplankton, respectively (Fig. 3a-d). OFX and RTM concentrations were significantly higher in zooplankton than all other antibiotics, and OTC and RTM concentrations had average values of $167.09 \mathrm{ng} \mathrm{g}^{-1}$ $\mathrm{dw}$ and $151.85 \mathrm{ng} \mathrm{g}^{-1} \mathrm{dw}$, respectively. Concentrations of OTC in the zooplankton ranged from 10.01 to $987.21 \mathrm{ng} \mathrm{g}^{-1} \mathrm{dw}$ (Fig. 3c), and the lowest and highest
RTM levels were $0.063 \mathrm{ng} \mathrm{g}^{-1} \mathrm{dw}$ and $779.68 \mathrm{ng} \mathrm{g}^{-1}$ dw (Fig. 3d). SMX, NFX and TC concentrations in zooplankton were also significantly higher than concentrations of SDZ, SPD, SCT, SMZ, TMP, CFX, LFX, OFX, ETM. The concentrations of SMX in the zooplankton ranged from 2.38 to $713.81 \mathrm{ng} \mathrm{g}^{-1} \mathrm{dw}$, with a mean of $89.16 \mathrm{ng} \mathrm{g}^{-1} \mathrm{dw}$ (Fig. 3a). Concentrations of NFX in zooplankton ranged from 1.63 to $396.03 \mathrm{ng} \mathrm{g}^{-1} \mathrm{dw}$, with a mean $64.34 \mathrm{ng} \mathrm{g}^{-1} \mathrm{dw}$ (Fig. 3b), and the lowest and highest TC levels were $0.069 \mathrm{ng} \mathrm{g}^{-1} \mathrm{dw}$ and $660.59 \mathrm{ng} \mathrm{g}^{-1}$ $\mathrm{dw}$, with a mean of $95.81 \mathrm{ng} \mathrm{g}^{-1} \mathrm{dw}$ (Fig. 3c). In this study, antibiotics in zooplankton were affected by phytoplankton biomass. For example, ETM, SDZ, RTM, SMX and OTC concentrations in zooplankton were significantly positively correlated with bacillariophyte biomass, and TMP concentrations in zooplankton increased when dinophyta biomass increased. SCT increased with chrysophyta biomass increasing, SMZ and OFX increased with euglenophyta increasing, 
Table 4. Mean antibiotic concentrations (ng g ${ }^{-1}$ dry weight) in zooplankton from four seasons in Yelang Lake Reservoir, P. R. China.

\begin{tabular}{|c|c|c|c|c|c|c|c|c|c|c|c|c|c|c|}
\hline & SDZ & SPD & SCT & SMX & SMZ & TMP & NFX & CFX & LFX & OFX & OTC & TC & ETM- ${ }_{2} \mathrm{O}$ & RTM \\
\hline Spring & 14.1 & 16.7 & 8.69 & 71.72 & 4.76 & 7.50 & 52.8 & 5.14 & 2.77 & 9.75 & 156.5 & 75.23 & 9.88 & 45.21 \\
\hline Summer & 11.9 & 25.7 & 5.63 & 98.40 & 4.41 & 2.06 & 31.6 & 40.9 & 1.80 & 17.87 & 133.6 & 77.98 & 63.89 & 224.3 \\
\hline Autumn & 23.1 & 11.4 & 9.50 & 51.69 & 4.13 & 5.99 & 90.3 & 22.2 & 6.42 & 4.95 & 238.9 & 180.1 & 23.02 & 190.9 \\
\hline Winter & 27.5 & 10.8 & 6.97 & 134.8 & 2.13 & 9.94 & 82.5 & 33.9 & 7.02 & 3.28 & 139.2 & 49.91 & 12.06 & 146.9 \\
\hline Min & 11.9 & 10.8 & 5.63 & 51.69 & 2.13 & 2.06 & 31.6 & 5.14 & 1.8 & 3.28 & 133.6 & 49.91 & 9.88 & 45.21 \\
\hline Max & 27.5 & 25.7 & 9.5 & 134.8 & 4.76 & 9.94 & 90.3 & 40.9 & 7.02 & 17.87 & 238.9 & 180.1 & 63.89 & 224.3 \\
\hline Average & 19.1 & 16.1 & 7.69 & 89.15 & 3.85 & 6.37 & 64.3 & 25.5 & 4.50 & 8.96 & 167.0 & 95.80 & 27.21 & 151.8 \\
\hline BAFs & 7.04 & 5.16 & 2.01 & 16.66 & 1.11 & 1.85 & 22.5 & 15.7 & 2.33 & 6.06 & 78.43 & 110.1 & 15.37 & 108.4 \\
\hline
\end{tabular}

and SPD and CFX were positively associated with cyanophyta and cryptophyta biomass (Fig. 4). The OTC concentration in zooplankton increased when Bosmina longirostris, D. dubium and rotifera increased (Fig. 5) and was positively associated with TN and TN (Fig. 6). The RTM concentration in zooplankton increased when $M$. leuckarti and D. pulex increased (Fig. 5) and was positively associated with $\mathrm{TP}, \mathrm{NO}_{2}-\mathrm{N}$ and $\mathrm{PO}_{4}-\mathrm{P}$ (Fig. 6). The SMX and NFX concentrations in zooplankton increased with $T$. brevifurcatus, T. mongolicus, and D. pulex (Fig. 5). However, the SMX concentration in zooplankton was negatively associated with $\mathrm{TN}, \mathrm{pH}$ and $\mathrm{COD}_{\mathrm{Mn}}$, and the SMX, RTM, CFX and ETM concentrations increased with increasing $\mathrm{PO}_{4}-\mathrm{P}$ and TP (Fig. 6). There was a positive correlation between TP and $\mathrm{PO}_{4}-\mathrm{P}(P<0.005, F=137.1)$. The TC concentration in zooplankton was significantly positively correlated with $\mathrm{TN}$ and $\mathrm{COD}_{\mathrm{Mn}}$ (Fig. 6). The other antibiotic concentrations (SDZ, SPD, SCT, SMZ, TMP, CFX, LFX, OFX, ETM) in zooplankton were lower, with means of $19.18 \mathrm{ng} \mathrm{g}^{-1}, 16.17 \mathrm{ng} \mathrm{g}^{-1}$, $7.69 \mathrm{ng} \mathrm{g}^{-1}, 3.85 \mathrm{ng} \mathrm{g}^{-1}, 6.36 \mathrm{ng} \mathrm{g}^{-1}, 25.56 \mathrm{ng} \mathrm{g}^{-1}$, $4.50 \mathrm{ng} \mathrm{g}^{-1}, 8.96 \mathrm{ng} \mathrm{g}^{-1}$, and $27.20 \mathrm{ng} \mathrm{g}^{-1}$ (Fig. 4a-d). SDZ, SMZ concentration in zooplankton significantly positively correlated with T. brevifurcatus, T. mongolicus, $D$. pulex biomass, and SPD with $P$. tunguidus, C. cornuta, TMP, ETM and OFX with P. tunguidus, C. cornuta, M. leuckarti, C. vicinus vicinus, CFX with $D$. pulex, LFX with T. brevifurcatus, T. mongolicus, Bosmina longirostris, D. dubium were significantly positively correlated, TC and SCT concentration in zooplankton had no significant correlation with zooplankton species (Fig. 5). SDZ, SPD, SCT, LFX and $\mathrm{SMZ}$ significantly increased with $\mathrm{TN}, \mathrm{COD}_{\mathrm{Mn}}$, and TMP with $\mathrm{NO}_{3}-\mathrm{N}, \mathrm{NH}_{3}-\mathrm{N}, \mathrm{DO}$ and $\mathrm{pH}$, OFX with DO, CFX and ETM with TP and $\mathrm{NO}_{2}-\mathrm{N}$ was significantly positively correlated (Fig. 6).

\section{Discussion}

The metals content in zooplankton is well established. However, the accumulation of many pollutants in zooplankton has not been considered; for example, antibiotic accumulation in zooplankton has received very little attention as the most studied pollutant today. At present, there are few studies on the bioaccumulation of antibiotics, and the existing results are mainly about the reports of residue detection methods of antibiotics in living bodies such as zoobenthos, fish, shrimp, etc. Residual reports of antibiotics in wildlife have focused more on fish and shrimp at high trophic levels. Zhang et al. [39] showed that twelve of 19 antibiotics were detected in shrimp samples with total concentrations ranging from 1.80 to $10.6 \mathrm{ng} \mathrm{g}^{-1}$ ww. Total antibiotic concentrations in the shrimp showed a negative correlation with their growth stage. Antibiotics in young shrimp with a low gross weight reached higher total concentrations (8.59-10.6 $\mathrm{ng} \mathrm{g}^{-1}$ ww) than middle-aged and adult shrimp. Even if the concentration of antibiotics in the water environment is not high, through biological concentration and food chain transfer, the concentration of antibiotics in the water environment may have a negative impact on ecological security and human health. In the study shown, a part of the antibiotics accumulation in zooplankton was very intensive, and the continuous exposure may lead to the accumulation of antibiotics in aquatic living bodies. Because the concentration of antibiotics was very much lower in the Yelang Lake Reservoir (the OTC, RTM and TC concentrations in the water had a lower mean value of $2.13 \mathrm{ng} \mathrm{L}^{-1}$, $1.40 \mathrm{ng} \mathrm{\textrm {L } ^ { - 1 }}, 0.87 \mathrm{ng} \mathrm{L}^{-1}$ respectively). However, antibiotics OTC, RTM, TC, SMX, NFX and NFX concentrations in the zooplankton had a higher value, with an average value of $167.09 \mathrm{ng} \mathrm{g}^{-1} \mathrm{dw}, 151.85 \mathrm{ng} \mathrm{g}^{-1}$ $\mathrm{dw}, 95.80 \mathrm{ng} \mathrm{g}^{-1} \mathrm{dw}, 89.85 \mathrm{ng} \mathrm{g}^{-1} \mathrm{dw}, 64.3 \mathrm{ng} \mathrm{g}^{-1} \mathrm{dw}$, respectively, and the study found that BAFs of antibiotics in zooplankton was very higher than others pollutants. The BAFs of OTC, RTM and TC, SMX and NFX were significantly higher, and the mean values of BAFs were 78.43 and 108.4 and $110.1,16.66$ and 22.5 , respectively (Table 2 and Table 4). Xie et al. [40] found that the mass ratio range of TC was from $26 \mathrm{ng} \mathrm{g}^{-1}$ to $49 \mathrm{ng} \mathrm{g}^{-1}$ in zoobenthos from Taihu Lake, and for plankton collected from Taihu Lake, TC displayed the highest median 

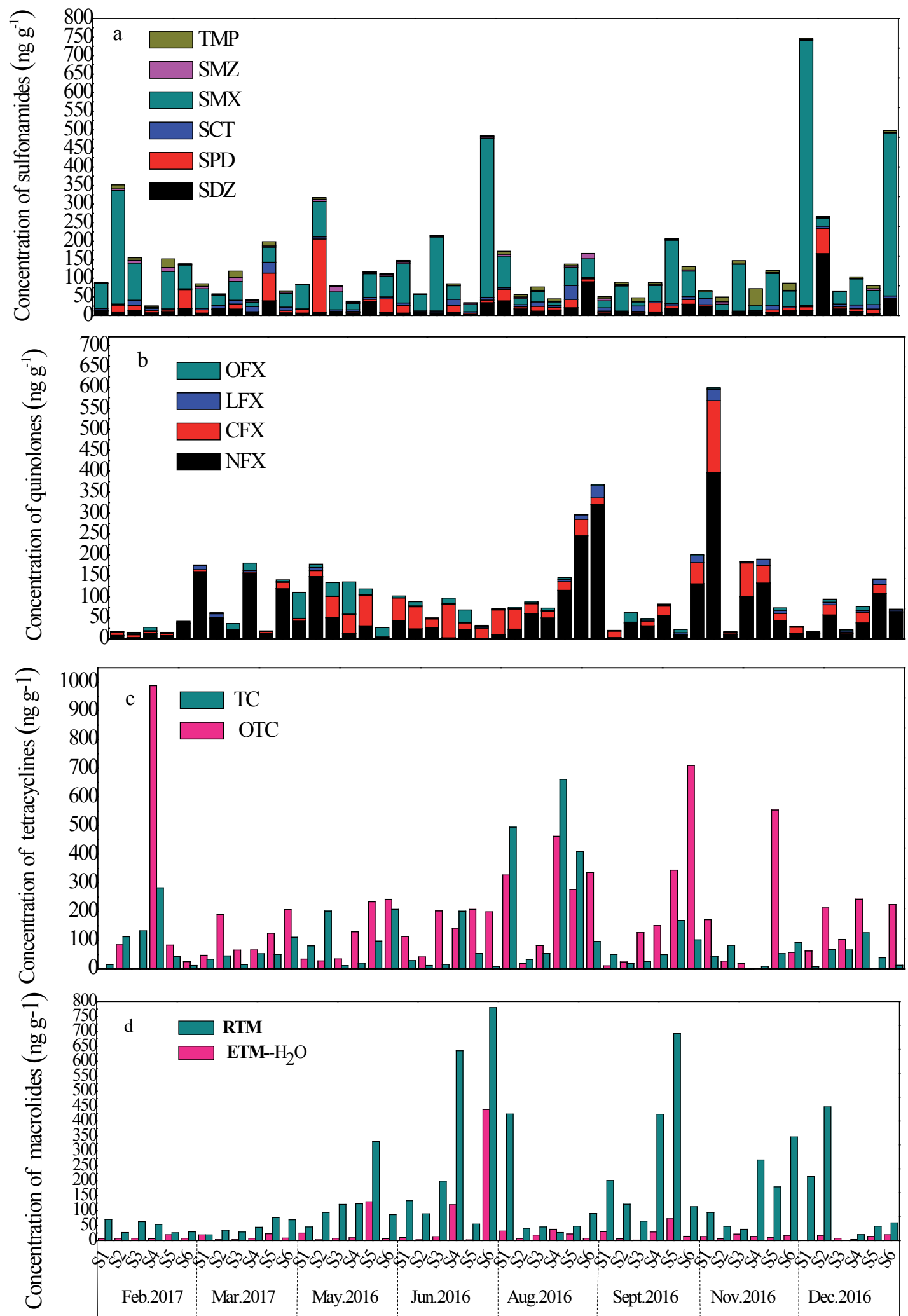

Fig. 3 Temporal-spatial variation of four kinds of antibiotics( trimethoprim (TMP), sulfamethazine (SMZ), sulfamethoxazole (SMX), sulfacetamide (SCT), sulfapyridine (SPD), sulfadiazine (SDZ), ofloxacin (OFX), lomefloxacin (LFX), ciprofloxacin (CFX), norfloxacin (NFX), oxytetracycline (OTC), tetracycline (TC), oxytetracycline (OTC), roxithromycin (RTM) and dehydroerythromycin (ETM-H2O) used in zooplankton in Yelang lake reservoir. 


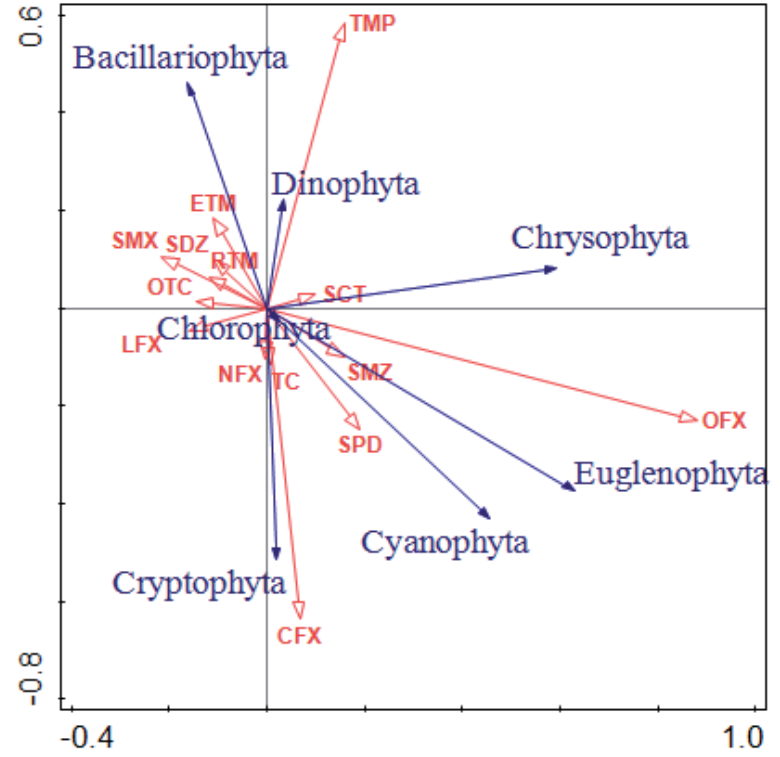

Fig. 4. CCA biplot representation of the impact of antibiotics Six SAs, the four FQs, two TCs, and two MLs in zooplankton and phytoplankton biomass from Yelang Lake Reservoir May. 2016 to Mar. 2017 using detrended canonical correspondence analysis.

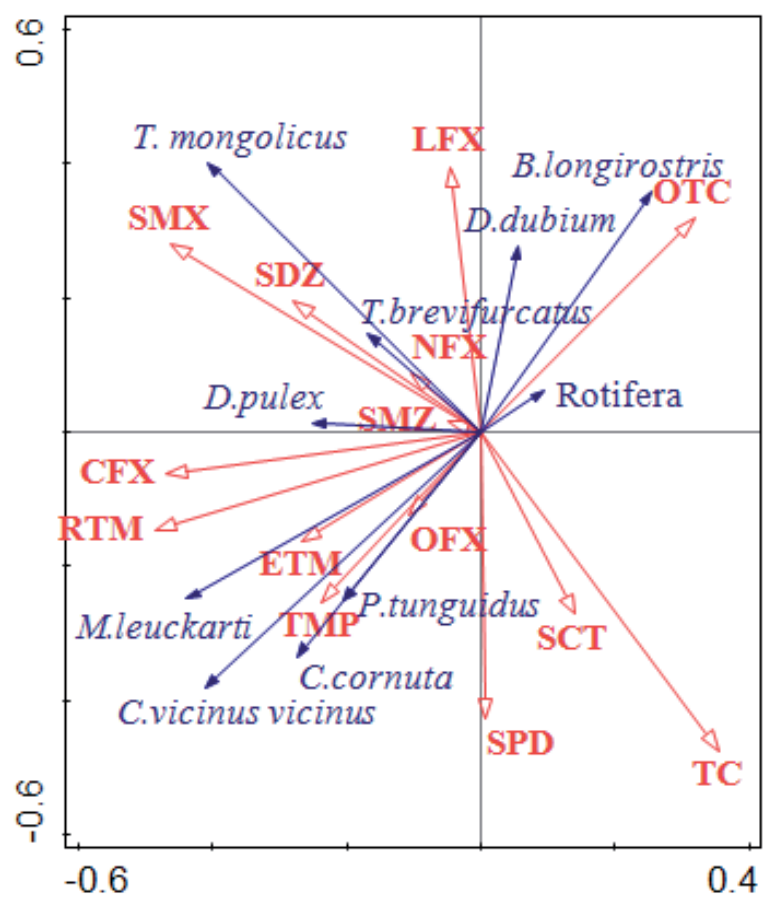

Fig. 5. CCA biplot representation of the impact of antibiotics Six SAs, the four FQs, two TCs, and two MLs in zooplankton and the biomass of different zooplankton species from Yelang Lake Reservoir May. 2016 to Mar. 2017 using detrended canonical correspondence analysis.

concentrations of 29 and $35 \mathrm{ng} / \mathrm{g}$ in phytoplankton and zooplankton, respectively. Our results were significantly similar to previous results; the antibiotics TC, OTC and RTM have intensive accumulation in zooplankton.

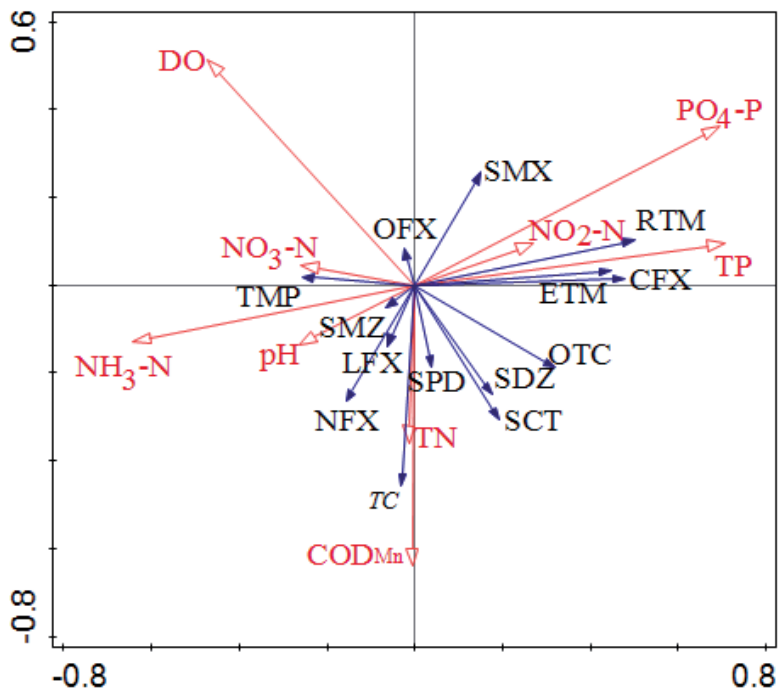

Fig. 6. CCA biplot representation of the impact of antibiotics Six SAs, the four FQs, two TCs, and two MLs in zooplankton and physicochemical property from Yelang Lake Reservoir May. 2016 to Mar. 2017 using detrended canonical correspondence analysis.

Du et al. [34] inspected a variety of antibiotics and the existence of their metabolites in wild fish samples in Bayou, and the results showed that ETM- $\mathrm{H}_{2} \mathrm{O}$ had the highest level of $6200 \mathrm{ng} \mathrm{g}^{-1}$ in H. plecostomus. However, our results showed that the ETM- $\mathrm{H}_{2} \mathrm{O}$ concentration in zooplankton was higher in summer (63.89 $\left.\mathrm{ng} \mathrm{g}^{-1}\right)$ and autumn (23.02 $\left.\mathrm{ng} \mathrm{g}^{-1}\right)$ than in the other two seasons. In this study, our results suggested that antibiotics with the highest concentrations in zooplankton also showed the strongest relationship to zooplankton bioaccumulation, similar to previous studies [40, 42]. In this study, we also showed that the concentrations of antibiotics in zooplankton were significantly correlated with zooplankton species. For example, the OTC concentration in zooplankton increased when Bosmina longirostris, D. dubium and rotifera increased, and the RTM concentration in zooplankton increased when $M$. leuckarti and D. pulex increased. SMX and NFX concentrations in zooplankton increased as T. brevifurcatus, T. mongolicus, and D. pulex increased. SDZ and SMZ concentrations in zooplankton were significantly positively correlated with $T$. brevifurcatus, T. mongolicus, and D. pulex biomass, and SPD with P. tunguidus, C. cornuta, TMP, ETM- $\mathrm{H}_{2} \mathrm{O}$ and $\mathrm{OFX}$ with P. tunguidus, C. cornuta, M. leuckarti, and C. vicinus vicinus, CFX with $D$. pulex, LFX with T. brevifurcatus, T. mongolicus, Bosmina longirostris, and D. dubium were significantly positively correlated (Fig. 5). Previous studies have shown that the concentrations of antibiotics in zooplankton were significantly correlated with zooplankton species [30]. The results also showed that selected antibiotic concentrations in zooplankton were species specific.

Kim et al. [41] found an acute toxicity effect of TMP and SMZ on Daphnia magna capricornutum. 
This result indicates that the effect of the antibiotics TMP and SMZ on Daphnia magna is not obvious. The main reason was that the concentration toxicity of antibiotics in zooplankton was slower. Wollenberger et al. [42] studied the acute and chronic toxicity of 9 kinds of veterinary antibiotics to Daphnia magna and found that environmentally related concentrations of antibiotics, such as metronidazole, olaquindox, streptomycin, sulfadiazine and tylosin, had no obvious effect on Daphnia magna. Our results also found that the SCT concentration in zooplankton was not significantly correlated with zooplankton species. In this study, our results suggested that the toxicity of antibiotics in zooplankton was very low, mainly because the portion of antibiotic concentrations in zooplankton was low. These antibiotic concentrations of SCT, SMZ, TMP, LFX, and OFX in zooplankton were lower, with a mean of $7.69 \mathrm{ng} \mathrm{g}^{-1} \mathrm{dw}, 3.85 \mathrm{ng} \mathrm{g}^{-1}$ $\mathrm{dw}, 6.36 \mathrm{ng} \mathrm{g}^{-1} \mathrm{dw}, 4.50 \mathrm{ng} \mathrm{g}^{-1} \mathrm{dw}$, and $8.96 \mathrm{ng} \mathrm{g}^{-1} \mathrm{dw}$, respectively. The BAFs of SCT, SMZ, TMP, LFX and OFX were significantly lower, and the mean values of BAFs were 2.01, 1.11, 1.85, 2.35 and 6.06, respectively. In this study, the antibiotics SCT, SMZ, TMP, LFX and OFX showed weak accumulation in zooplankton. Our results suggested that the lower concentrations of SCT, SMZ, TMP, LFX, and OFX in zooplankton were affected mainly by the phytoplankton biomass of the reservoir. For example, the OTC, RTM and SMX concentrations in zooplankton were significantly positively correlated with bacillariophyte biomass, diatom biomass was higher than other phytoplankton biomasses in the reservoir, and its proportion averaged more than $53.38 \%$. A previous study showed that diatoms were the best food for zooplankton [30]. The results showed higher concentrations of OTC, RTM and SMX in zooplankton, mainly because diatoms can be assimilated by zooplankton, and lower SCT, SMZ and OFX concentrations in zooplankton were significantly associated with lower chrysophyta and euglenophyte biomass (Fig. 2a).

A previous study showed that antibiotics can enter the water and land environment through various pathways, such as the discharge of municipal sewage, manufacturing industry, animal husbandry, and urban centres. Discharge with high $\mathrm{TN}, \mathrm{NO}_{3}-\mathrm{N}, \mathrm{NH}_{3}-\mathrm{N}$, $\mathrm{NO}_{2}-\mathrm{N}, \mathrm{COD}, \mathrm{DO}, \mathrm{pH}, \mathrm{TP}$ and $\mathrm{PO}_{4}-\mathrm{P}$ and antibiotic concentrations. Therefore, antibiotics in zooplankton also affected $\mathrm{TN}, \mathrm{NO}_{3}-\mathrm{N}, \mathrm{NH}_{3}-\mathrm{N}, \mathrm{NO}_{2}-\mathrm{N}, \mathrm{COD}, \mathrm{DO}$, $\mathrm{pH}$, and TP. In this study, the bioaccumulation of antibiotics was most strongly affected by $\mathrm{TN}, \mathrm{NO}_{3}-\mathrm{N}$, $\mathrm{NH}_{3}-\mathrm{N}, \mathrm{NO}_{2}-\mathrm{N}, \mathrm{COD}, \mathrm{DO}, \mathrm{pH}, \mathrm{TP}$ and $\mathrm{PO}_{4}-\mathrm{P}$. For example, the OTC concentration in zooplankton was positively associated with $\mathrm{TN}$ and TP. RTM was positively associated with $\mathrm{TP}, \mathrm{NO}_{2}-\mathrm{N}$ and $\mathrm{PO}_{4}-\mathrm{P}$. SMX and NFX were positively associated with $\mathrm{TN}, \mathrm{pH}$ and $\mathrm{COD}_{\mathrm{Mn}}$. The SMX concentration in zooplankton was negatively associated with $\mathrm{TN}, \mathrm{pH}$ and $\mathrm{COD}_{\mathrm{Mn}}$ and positively associated with $\mathrm{PO}_{4}-\mathrm{P}, \mathrm{NO}_{2}-\mathrm{N}$, and TP. The TC concentration in zooplankton was significantly positively correlated with $\mathrm{TN}$ and $\mathrm{COD}_{\mathrm{Mn}}$. SDZ, SPD, SCT, LFX and SMZ significantly increased with TN, $\mathrm{COD}_{\mathrm{Mn}}$, and TMP with $\mathrm{NO}_{3}-\mathrm{N}, \mathrm{NH}_{3}-\mathrm{N}, \mathrm{DO}$ and $\mathrm{pH}$, OFX with DO, CFX and ETM with TP and $\mathrm{NO}_{2}-\mathrm{N}$ was significantly positively correlated. In this study, our results suggested that domestic sewage discharge can increase the risk of antibiotic concentrations in zooplankton. The main reason domestic sewage can enhance phytoplankton biomass blooms, enhance zooplankton food, and indirectly affect the bioaccumulation of antibiotics. Antibiotics in estuaries and groundwater generally originate from aquaculture wastewater and untreated/treated domestic sewage. Most of the detected antibiotics posed no risk to the environment. Ciprofloxacin and enrofloxacin found in water may present high ecological and resistance risks, while the two antibiotics observed to accumulate in fish may pose a considerable risk to human health through diet consumption [43]. Our research results suggest that SDZ, NFX, LFX, SCT, TMP, OTC, SPD, TC, and CFX concentrations may be significantly affected by water metals. Some scholars have shown that tetracycline in the environment has a significant inhibiting effect on the movement of Daphnia magna, and the inhibition concentration is $617.2 \mathrm{mg} \mathrm{L}^{-1}$. Other studies have shown that the effect of tetracycline antibiotics on plants has two aspects of transcription and translation, while quinolone antibiotics affect chloroplast gene replication in aquatic plants [39]. OTC has the same chemical structure as tetracycline, and it is obvious that they have obviously similar effects on zooplankton. Therefore, this study supposes that higher concentrations of OTC may promote rather than inhibit the activity of zooplankton.

\section{Conclusions}

Antibiotic (OTC, RTM and TC) accumulation in zooplankton was very intense. Antibiotic (OTC, RTM and TC) concentrations were much lower in the Yelang Lake Reservoir, and the BAFs of OTC, RTM and TC were significantly higher than the BAFs of other antibiotics. The antibiotic concentrations of SCT, SMZ, TMP, LFX, and OFX in zooplankton were lower, and the BAFs of SCT, SMZ, TMP, LFX and OFX were significantly lower. Concentrations of OTC, RTM and SMX in zooplankton were higher mainly because higher diatom biomass can be assimilated by zooplankton, and lower SCT, SMZ and OFX concentrations in zooplankton were significantly lower chrysophyta and euglenophyte biomass. The concentration of antibiotics in zooplankton was significantly correlated with zooplankton species. The antibiotic OTC concentration in zooplankton increased when Bosmina longirostris, D. dubium and rotifera increased, and the RTM concentration in zooplankton increased when M. leuckarti and D. pulex increased. SMX and NFX concentrations in zooplankton increased as T. brevifurcatus, T. mongolicus, and D. pulex increased. 
SDZ and SMZ concentrations in zooplankton were significantly positively correlated with $T$. brevifurcatus, T. mongolicus, and D. pulex biomass. Antibiotics that can enter the water environment pathways were mainly the discharge of municipal sewage, manufacturing industry wastes and fish cage culture, and with a large amount of TN and TP. Antibiotic (OTC, TC and RTM) concentrations in zooplankton were positively associated with TN and TP. TP and TN concentrations may increase the risks posed to aquatic ecosystems because increases in TP and TN promote selective phytoplankton taxon growth and different antibiotic bioaccumulation in zooplankton with food transfer to humans.

\section{Acknowledgments}

This work was supported by National Key Research and Development Program (2016YFC0502607); Programme of Introducing Talents of Discipline to Universities (D17016); National Top Discipline Construction Project of Guizhou Province (No.125 2019).

\section{Author Contributions}

N.-Y, S.-L. and K.-X. designed and wrote the research; N.-Y and S.-L. wrote the paper; K.-X. and T. $-Z$ performed the experiments and data analysis.

\section{Competing Interests}

The authors declare no competing interests.

\section{References}

1. WILSON D.C. Potential urban runoff impacts and contaminant distributions in shoreline and reservoir environments of Lake Havasu, southwestern United States. Science of the Total Environment, 621, 95, 2018.

2. TANG J.P., WANG S., FAN J.J., LONG S.X., WANG L., TANG C., TAM N.F., YANG Y. Predicting distribution coefficients for antibiotics in a river water-sediment using quantitative models based on their spatiotemporal variations. Science of the Total Environment, 655, 1301, 2019.

3. KARAOUZAS I., KAPETANAKI N., MENTZAFOU A., KANELLOPOULOS, T.D., SKOULIKIDIS, N. Heavy metal contamination status in greek surface waters: A review with application and evaluation of pollution indices. Chemosphere, 128, 192, 2020.

4. WU J., LU, J., ZHANG, C., ZHANG, Y., XU, J. Pollution, sources, and risks of heavy metals in coastal waters of china. Human and Ecological Risk Assessment, 26, 2011, 2019.

5. PETUKHOY V.I., PETROVA E.A., LOSEV O.V. Water pollution by heavy metals and oil products in uglovoi bay in february 2010-2016. Water Resources. 46, 103, 2019.
6. LIU X., LU S., WEI G., XI B., WANG W. Antibiotics in the aquatic environments: a review of lakes, China. Science of the Total Environment, 627, 1195, 2018.

7. BEN Y.J., FU C.X., HU M., LIU L., WONG M.H., ZHENG C,M., Human health risk assessment of antibiotic resistance associated with antibiotic residues in the environment: A review. Environmental Research, 169, 483, 2019.

8. KUMAR M., JAISWA S., SODHI K.K., SHREE P., SINGH D.K., AGRAWAL P.K., SHUKLA P., Antibiotics bioremediation: perspectives on its ecotoxicity and resistance. Environment International, 124, 448, 2019.

9. BINH V.N., DANG N., ANH N.T.K., KY L.X., THAI P.K., Antibiotics in the aquatic environment of Vietnam: Sources, concentrations, risk and control strategy. Chemosphere, 197, 438, 2018.

10. ECONOMOU V., GOUSIA P. Agriculture and food animals as a source of antimicrobial resistant bacteria. Infection and Drug Resistance, 8, 49, 2015.

11. XIN Y., A Study on Toxicity and Antimicrobial Resistance of Antibiotic Wastewater and Control Technologies. Tsinghua University, Beiing, 2014 [In Chinsese].

12. KAN J., ZHU X.X., WANG T.Y., LU R.Z., SPENCER P.S., Chinese patient demand for intravenous therapy: a preliminary survey. The Lancet, 386, S61, 2015.

13. LIU X.H., LU S.Y., GUO W., XI B.D., WANG W.L., Antibiotics in the aquantic environments: A review of lake, China. Science of the Total Environment, 627, 1195, 2018.

14. ZHANG Q.Q., Emission Estimation, Multimedia Fate Modeling and Risk Assessment of Typical Emerging Pollutants at River Basin Scale in China. Guangzhou, Guangzhou Institute of Geochemistry of Chinese Academy of Sciences 2015 [In Chinsese].

15. UCHIDA K., KONISHI Y., ZHAO J.L. Comprehensive evaluation of antibiotics emission and fate in the river basins of China: source analysis, multimedia modeling, and linkage to bacterial resistance. Environmental Science and Technology, 49, 6772, 2015.

16. QIAO M., YING G.G., SINGER A.C., ZHU Y.G., Review of antibiotic resistance in China and its environment. Environ. Int., 110, 160, 2017.

17. CARVALHO I.T., SANTOS L., Antibiotics in the aquatic environments: a review of the European scenario. Environ. Int. 94, 736, 2016.

18. YAO L.L., WANG Y.X., TONG L., DENG Y.M., ZHAO $\mathrm{K}$. Occurrence and risk assessment of antibiotics in surface water and groundwater from different depths of aquifers: a case study at Jianghan Plain, central China. Ecotoxicology and Environmental Safety, 135, 236, 2017.

19. LIU X., STEELE J.C., MENG X.Z. Usage, residue, and human health risk of antibiotics in Chinese aquaculture: A review. Environmental Pollution, 223, 161, 2017.

20. LI Q., GAO J., ZHANG Q., LIANG L., TAO H. Distribution and risk assessment of antibiotics in a typical river in North China Plain. Bulletin of Environmental Contamination and Toxicology, 98, 478, 2017.

21. AHMED M.J., ISLAM M.A., ASIF M., HAMEED B.H., Human hair-derived high surface area porous carbon material for the adsorption isotherm and kinetics of tetracycline antibiotics. Bioresour. Technol. 243, 778, 2017.

22. HE D.C., XU Z.C., WU G.Y., QIU J.R., QIN G.J., Advance in heat stress mechanism effect in animals. Prog. Vet. Med. 32, 98, 2011 [In Chinsese].

23. HU J., SHI J., CHANG H., LI D., YANG M., KAMAGATA Y., Phenotyping and genotyping of antibiotic-resistant 
Escherichia coli isolated from a natural river basin. Environ. Sci. Technol, 42, 3415, 2008.

24. SEVILLASNCHEZ D., SOYMUNER, D., SOLER PORCAR, N., Usefulness of macrolides as antiinflammatories in respiratory diseases. Arch. Bronconeumol. 46, 244, 2010.

25. HE X.T., WANG Q., NIE X.P., YANG Y.T., CHENG Z. Residues and health risk assessment of sulfonamides in sediment and fish from typical marine aquaculture regions of Guangdong province, China. Environmental science, 35, 2728, 2014 [In Chinsese].

26. FANG Y.L. Fisheries in Guizhou: dismantling cage, not dismantling industry. Contemporary Guizhou. 24, 27, 2019 [In Chinsese].

27. CHEN B. Hydrological and Hydrochemical characteristics of the observation wells at puding hydropower station and their potential risk assessment. Earth and Environment, 42, 540, 2014 [In Chinsese].

28. LONG S.X., YANG Y., XIA P.H., CHEN C., LIU Z.W., MA J.R., DONG X., HE T.R., YUN G., Accumulation of metals in zooplankton from karst plateau reservoirs with different eutrophic status in Guizhou Province, P.R. China. Crustacean, 89, 537, 2016.

29. LONG S.X., HAMILTON P.B., YANG Y., WANG S., HUANG W.D., CHEN C., TAO R., Differential bioaccumulation of mercury by zooplankton taxa in a mercury-contaminated reservoir Guizhou China. Environmental Pollution, 239, 147, 2018.

30. LONG S.X., HAMILTON B.P., CHEN C., Effect of algal and bacterial diet on heavy metal bioaccumulation in zooplankton from the Pearl River, South China. Science of The Total Environment, 675, 151, 2019.

31. YE Z., WEINBERG H.S., MEYER M.T., Trace analysis of trimethoprim and sulfonamide, macrolide, quinolone, and tetracycline antibiotics in chlorinated drinking water using liquid chromatography electrospray tandem mass spectrometry[J]. Analytical Chemistry, 79, 1135, 2007.

32. HU H.J., WEI Y.X.,The Freshwater Algae of China Systematics, Taxonomy and Ecology. Science Press, Beijing, 2006.

33. XIE Z.X., LU G.H., YAN Z.H., LIU J.C., WANG P.F., WANG Y.H., Bioaccumulation and trophic transfer of pharmaceuticals in food webs from a large freshwater lake. Environmental Pollution, 222, 356, 2017.
34. DU B., HADDAD, S.P., LUEK A., SCOTT W.C., SAARI, G.N., BURKET, S.R., BREED, C.S., KELLY, M., BROACH, L., RASMUSSEN, J.B., CHMBLISS, C.K., BROOKS, B.W., Bioaccumulation of human pharmaceuticals in fish across habitats of fish across habitats of a tidally influenced urban bayou. Environmental Toxicology and Chemistry, 35, 966, 2016.

35. WANG J.J. Freshwater Rotifera. In: Fauna Sinica, Science Press, Beijing, 283, 1961 [In Chinsese].

36. JIANG Y.Z., CHU N.S., CRUSTACEA. Freshwater cladocerans. In: Fauna Sinica, Science Press, Beijing, 294, 1979 [In Chinsese].

37. SHEN C.J., SONG D.X., Freshwater Copepoda: Calanoida. In: Fauna Sinica, Crustacea, Science Press, Beijing, 450, 1979 [In Chinsese].

38. Environment Canada, Canadian guidance framework for the management of phosphorus in freshwater systems. National Guidelines and Standards Ofce, Water Policy and Coordination Directorate, Environment Canada, Report, 1-8, 64, 2004

39. ZHANG R.L., PEI J.Y., ZHANG R.J., WANG S.P., ZENG W.B., HUANG D.L., WANG Y., ZHANG Y.Y., WANG Y.H., YU K.F. Occurrence and distribution of antibiotics in mariculture farms, estuaries and the coast of the Beibu Gulf, China: Bioconcentration and diet safety of seafood. Ecotoxicology and Environmental Safety, 154, 27, 2018.

40. XIE Z.X., LU G.H., YAN Z.H., LIU J.C., WANG P.F., WANG Y.H., Bioaccumulation and trophic transfer of pharmaceuticals in food webs from a large freshwater lake. Environmental Pollution, 222, 356, 2017.

41. KIM Y., CHOI, K. JUNG J. Aquatic toxicity of acetaminophen, carbamazepine cimetidine, diltiazem and six major sulfonamides, and their potential ecological risks in. Korea. Environment International, 33, 370, 2007.

42. WOLLENBERGER L., HALLING S., RENSEN B., KUSKK O. Acute and chronic toxicity of veterinary antibiotics to Daphnia magna. Chemosphere, 40, 723, 2000.

43. HAN Q.F., ZHANG X.R., WANG X.L., SONG C., WANG S.G., Distribution, combined pollution and risk assessment of antibiotics in typical marine aquaculture farms surrounding the Yellow Sea, North China. Environment International, 138, 105551, 2020. 